Liu et al., Afr J Tradit Complement Altern Med. (2014) 11(2):273-276

\title{
STUDY ON THE ANTI-DEPRESSANT EFFECT OF CHAIHU GUIZHI DECOCTION AND ITS MECHINISIMS OF ACTIONS
}

\author{
Yu Liu ${ }^{1}$, Chunmei $\mathrm{Cao}^{2 *}$, Haijun Ding ${ }^{3}$ \\ ${ }^{1}$ Department of Psychology, Peking University, Beijing 100871, China. ${ }^{2}$ Department of Physical Education, Tsinghua \\ University, Beijing 100084, China. ${ }^{3}$ Fusong Hospital of Traditional Chinese Medicine, Fusong 134500, China. \\ *E-mail: pingkew32@126.com
}

\begin{abstract}
Background: Chaihu Guizhi has the effects of treating exogenous wind-cold; alternating episodes of chills and fever; noisy nose and retching; strong headache; chest and rib-side pain, and fullness, in clinical practice, it can also be used for the treatment of depression. The objective of this paper was to study the anti-depressant effect of Chaihu Guizhi decoction and its mechanism.

Materials and Methods: The anti-depressant effect of Chaihu Guizhi decoction was investigated by forced swimming test, tail suspension test and antagonism of reserpine hypothermia test in mice.

Results: Chaihu Guizhi decoction could significantly shorten immobility time of mice in the forced swimming test, and dose-dependence significantly shorten immobility time of mice in the tail suspension test, after administration of 50,100, and $200 \mathrm{mg} / \mathrm{kg}$, Chaihu Guizhi decoction, a significant dose-dependent antagonism of reserpine-induced hypothermia was observed in mice of each treatment group.

Conclusion: Chaihu Guizhi decoction has an antidepressant effect.
\end{abstract}

Key words: Chaihu Guizhi Decoction; forced swimming; tail suspension test.

\section{Introduction}

Chaihu Guizhi decoction originated from the Chinese medical classic "Treatise on Cold-induced Febrile Diseases", "Chaihu Guizhi decoction treats patients who have typhoid fever between 6-7, days experiencing fever, mild aversion to cold, vexing pain of the limb joints, mild retching, and propping bind below the heart, with exterior syndrome still present." This formula is the combined prescription of Xiao Chai Hu decoction, and Gui Zhi decoction, which consists of nine herbs of Chai Hu, Gui Zhi, Huang Qin, Ren Shen, Gan Cao, Ban Xia, Bai Shao, Da Zao and Sheng Jiang, and has the effects of treating exogenous wind-cold, alternating episodes of chills and fever, noisy nose and retching, strong headache, chest and rib-side pain, and fullness; in clinical practice, it can also be used for the treatment of depression (Meng et al., 2003; Dong et al., 2007). This study investigated its anti-depressant pharmacological effects and mechanism by an animal model of behavioral despair.

\section{Materials and Methods \\ Animals}

Kunming mice, male, $(20 \pm 2)$ g, purchased from China Medical University, kept at room temperature, fed ad libitum, adaptive feeding for four days. All experimental procedures were approved by the Animal Research Ethics Committee.

\section{Drugs and reagents}

All the medical materials in the prescription of Chaihu Guizhi decoction were purchased from the market, which were identified as: the dried roots of Bupleurum chinense DC. of family Umbelliferae (Chai Hu), dried twigs of Cinnamomum cassia Prel of family Lauraceae (Gui Zhi), dried roots of Scutellaria baicalensis of family Labiatae (Huang Qin), dried roots of Panax ginseng C. A. Mey of family Araliaceae (Ren Shen), dried roots and rhizomes of Glycyrrhiza uralensis Fisch of family Leguminosae (Gan Cao), dried tubers of Pinellia ternate (Thunb) Breit of family Araceae (Ban Xia), dried roots of Paeonia lactiflora Pall., family Ranunculaceae (Bai Shao), dried ripe fruits of Ziziphus jujuba Mill of family Rhamnaceae (Da Zao), and dried tubers of Zingiber offcinale Rosc., of family Zingiberaceae (Sheng Jiang). The above nine medicinal materials were soaked in water for 30min., decocted for 30 40min., separately, filtered, and the filtrates were combined and concentrated to test concentrations for later use. Fluoxetine hydrochloride tablets (Changzhou Siyao Pharmaceutical Co., Ltd.), reserpine (Tianjin KingYork Amino Acid Co., Ltd.)

\section{Animal grouping and administration methods}

The mice were randomly divided into five groups: blank control group (distilled water), Chaihu Guizhi decoction treatment groups and positive control group (fluoxetine $20 \mathrm{mg} / \mathrm{kg}$ ), each group contained ten mice; dose volume was all $0.2 \mathrm{ml} / 10 \mathrm{~g}$. The mice in each group were administered intra-gastrically at 8:00am daily for 7, consecutive days; the test was performed on the 7th day, $1 \mathrm{hr}$ after administration.

\section{Open field test (Archer, 1973)}

90min., after the last administration, the mice were placed in a cylindrical container $30 \mathrm{~cm}$ in diameter and $20 \mathrm{~cm}$ in height with bottom divided into 19 , equal sections, and the walking routes and the number of grid crossings of mice within $2 \mathrm{~min}$., in each group were compared. 
http://dx.doi.org/10.4314/ajtcam.v11i2.7

\section{Forced swimming test (Porsolt et al., 1977)}

90min., after the last administration, the mice were placed in a cylindrical glass tank $\left(20 \mathrm{~cm}\right.$ high x $14 \mathrm{~cm}$ diameter); with water temperature of $25^{\circ} \mathrm{C}$, after $4 \mathrm{~min}$., of swimming, the immobility time of mice within the $4 \mathrm{~min}$, was observed cumulatively. Immobility refers to the state in which the mouse stops struggling in the water, or remains floating.

Tail suspension test (Steru et al., 1985)

$1 \mathrm{hr}$, after the last administration, the tail of mice about $2 \mathrm{~cm}$ from the tip was attached to a horizontal holder, so that the mice were hung upside-down with their heads approximately $5 \mathrm{~cm}$, away from the table, 2 , mice were suspended each time, which were separated by a partition. Suspension time was 6 min., and the cumulative immobility time of mice within the last 4 min., was recorded.

\section{Reserpine antagonism test (Zheng et al., 2005)}

$1 \mathrm{hr}$, after the last administration, the mice were intra-peritoneally injected with $5 \mathrm{mg} / \mathrm{kg}$, reserpine, $2 \mathrm{hr}$, later, rectal temperature was measured with a digital thermometer, and differences in the change of rectal temperature between the treatment group, control group and normal control group were compared.

\section{Statistical analysis}

Analysis of variance on the experimental data was performed using SPSS 13, software. Significance criterion $\alpha=0.05$.

\section{Results \\ Open field test}

The results are shown in Fig. 1, the locomotor activity of mice in each treatment group (50, 100, and 200mg/kg), did not change significantly compared with the control group, indicating that the drug had no significant effect on locomotor activity in mice.

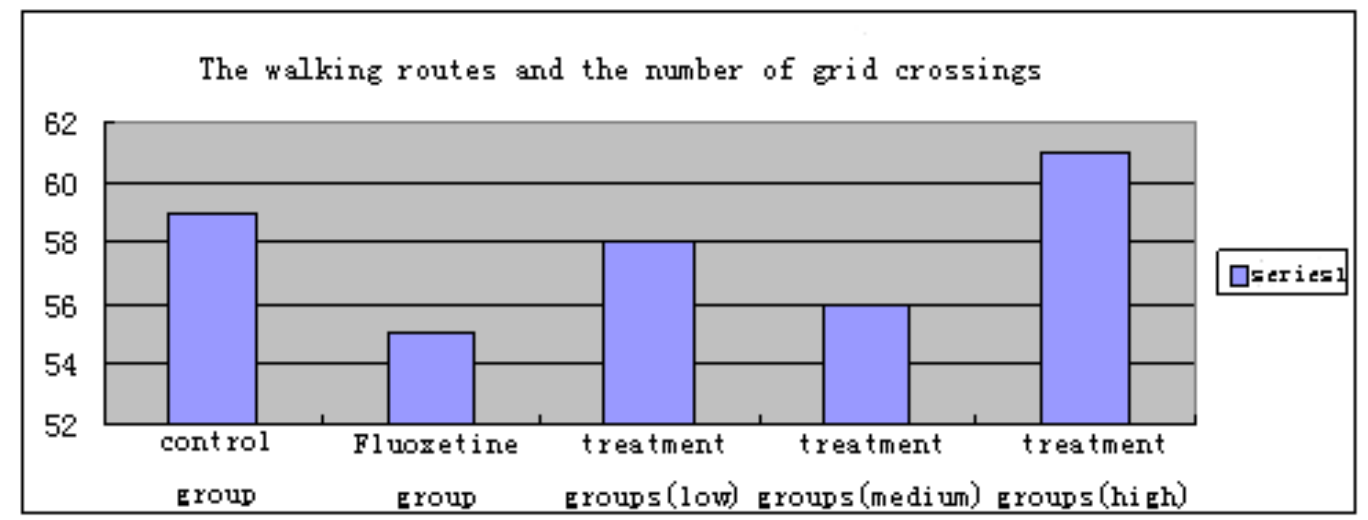

Figure 1: Effect of Chaihu Guizhi Decoction on locomotor activity in mice

\section{Forced swimming test}

The immobile state of mice present in the forced swimming model reflects the despair behavior of animals, the forced swimming test results showed that, Chaihu Guizhi decoction could significantly shorten the immobility time of mice in the forced swimming test, after administration of different doses of Chaihu Guizhi decoction to the animal model, varying degrees of antidepressant effects were observed in each group (Figure 2).

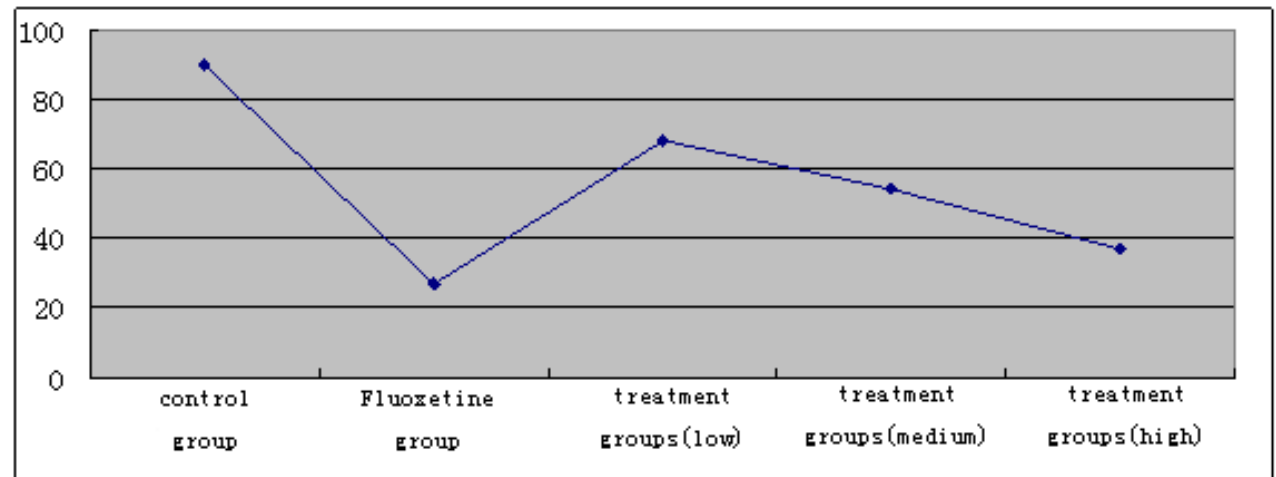

Figure 2: Effect of Chaihu Guizhi Decoction on immobility time in forced-swimming mice 


\section{Effect on tail suspension test}

The test results are shown in Fig. 3. The immobile state of mice present in the tail suspension model also reflects the despair behavior of animals, compared with the blank control group, the Chaihu Guizhi Decoction three dose groups could significantly shorten the immobility time of tail-suspended mice in a dose-dependent manner.

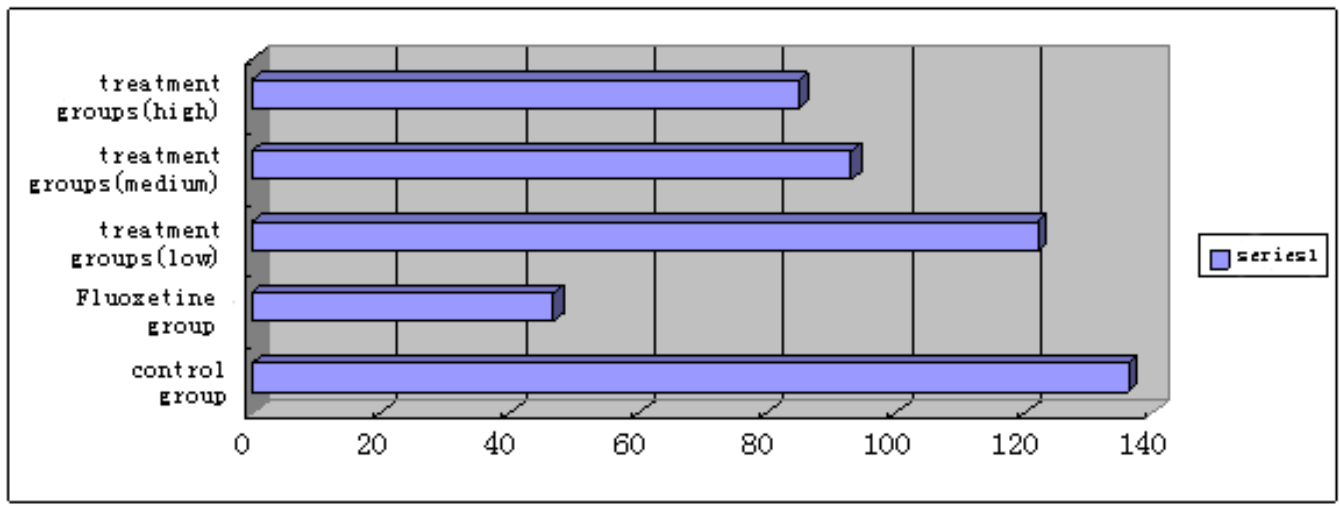

Figure 3: Effect of Chaihu Guizhi Decoction on immobility time in tail-suspended mice

\section{Antagonism of reserpine-induced hypothermia}

After administration of 50,100 , and $200 \mathrm{mg} / \mathrm{kg}$, Chaihu Guizhi decoction, the results of each treatment group showed a significant dose-dependent antagonism of reserpine-induced hypothermia in mice.

Table 1: Effect of Chaihu Guizhi Decoction on reserpine-induced hypothermia in mice $(x \pm s, n=10)$

\begin{tabular}{|c|c|c|c|c|}
\hline \multicolumn{3}{|l|}{ Group } & Dose $(\mathrm{mg} / \mathrm{kg})$ & Mouse body temperature $\left({ }^{\circ} \mathrm{C}\right)$ \\
\hline Normal control gl & oup & & & $36.9 \pm 0.95^{* *}$ \\
\hline Reserpine group & & & & $32.9 \pm 0.38$ \\
\hline Fluoxetine group & & & 20 & $33.3 \pm 0.56 *$ \\
\hline $\begin{array}{l}\text { Chaihu Guizhi } \\
\text { (low) }\end{array}$ & Decoction & group & 50 & $33.7 \pm 1.24 * *$ \\
\hline $\begin{array}{l}\text { Chaihu Guizhi } \\
\text { (medium) }\end{array}$ & Decoction & group & 100 & $33.8 \pm 1.53^{* *}$ \\
\hline $\begin{array}{l}\text { Chaihu Guizhi } \\
\text { (high) }\end{array}$ & Decoction & Group & 200 & $34.8 \pm 1.73^{* *}$ \\
\hline
\end{tabular}

Note: Comparison with the control group, ${ }^{*} \mathrm{P}<0.05$, ${ }^{* *} \mathrm{P}<0.01$.

\section{Discussion}

Depression is a neurological disorder whose main symptoms include low mood, difficulty in concentrating, spiritual malaise, irritability, retardation of thinking, reduced verbal and physical actions, loss of interest in daily life, loss of appetite, etc. (Ruo et al., 2000). The factors causing depression include: genetic factors, physical factors, the function of central neurotransmitters, metabolic abnormalities, mental factors, etc. There are many etiological factors for depression, which is related to genetic, environmental, social and other factors. Its pathogenesis is not yet fully understood, some scholars have studied its pathogenesis, which include monoamine neurotransmitter theory, endocrine theory, signal transduction pathway theory, immune abnormality theory, and the newly proposed neural plasticity i.e. neurotrophic hypothesis, etc. (Mao et al.,2007; Yatham et al., 2000).

Chinese medicine believes that the heart governs blood vessels, and houses the mind. Insufficient congenital endowment and weakness of qi and blood are the underlying determinants of the incidence of depression, and are the key causes of depressive illness. If congenital endowment is adequate, and yin and yang are in equilibrium, it will only be a momentary emotional discomfort when affected by external stimuli, which can be recovered through self-regulation without treatment; on the contrary, if congenital endowment is deficient, plus failure and other emotional injuries are suffered, yin and yang will be disharmonized, leading to the onset of depression. Emotional stress and discomfort are the external predisposing factors for the incidence of depression, the combination of internal and external etiological factors can easily lead to the onset of depressive illness.

This study investigated the effect of Chaihu Guizhi decoction on animal model of depression, and its anti-depressant mechanism of action. The forced swimming model and tail suspension test are classic anti-depressant models, which reflect the despair behavior of animals, and can simulate the depressive state of humans. Different doses of Chaihu Guizhi decoction can significantly shorten the immobility time of mice, which showed relatively strong anti-depressant effects. In the reserpine-induced hypothermia antagonism test in mice, the three doses of Chaihu Guizhi decoction can effectively inhibit the reserpine-induced hypothermia in mice; there were significant differences in the data between the groups, which were consistent with its clinical effectiveness in the treatment of depression. 
Liu et al., Afr J Tradit Complement Altern Med. (2014) 11(2):273-276

http://dx.doi.org/10.4314/ajtcam.v11i2.7

\section{Acknowledgement}

The work was supported by Research on the impact of different load and unsteady on the coordination of body trunk, and lower limb, Independent scientific research plan, Ministry of Education.

\section{References}

1. Archer, J. (1973). Tests for Emotionality in Rats and Mice: A review. Animal Behaviour, 21: 205-235.

2. Dong, F. Q., Zhu, W. E., Xie, W. T., Chen, Z. Y., Li, T., and Wu, J. J. (2007) Effect of Chaihu Plus Longgumuli Decoction on Psychological Stress Reaction in Rats. Journal of Zhejiang Chinese Medical University, 31(1): 58-61.

3. Mao, Q. Q., and Huang, Z. (2007). Research Progress on Therapeutic Mechanism of Depression Treated by Traditional Chinese Medicine. China Journal of Chinese Materia Medica, 32(10): 778-088.

4. Meng, H. B., Qu, R., and Ma, S. P. (2003). Anti-depressant Effect of Caihujialonggumuli Decoction. Pharmacology and Clinics of Chinese Materia Medica, 19(1): 3-6.

5. Porsolt, R. D., Bertin, A., and Jalfre, M. (1977). Behavioural Despair in Mice: A Primary Screening Test for Antidepressants. Archives Internationales de Pharmacodynamie et de Therapie. 229: 327-330.

6. Ruo, L., and Tan, R. X. (2000). Progress in Pharmacological Research of Antidepressant Drugs. Progress in Pharmacological Researches, 75-82.

7. Schelkunov, E. L. (1968). Apomorphine Hypothermia in Mice as the Test for Differentiation of Antidepressants and Cholinolytics. Experimental Studies of Antidepressants, 62-77.

8. Steru, L., Chermat, R., Thierry, B., and Simon, P. (1985). The Tail Suspension Test: A New Method for Screening Antidepressants in Mice. Psychopharmacology, 85(3): 367-370.

9. Yatham, L. N., Liddle, P. F., Sossi, V., Erez, J., Vafai, N., Lam, R. W., and Blinder, S. (2000). Brain Serotonin Receptor in Major Depression: A Positron Emission Tomography Study. Areh-gen Psychiatry, 57: 85-28.

10. Zheng, L. F., and Ming, L. (2005). The Study and Application of Animal Models of Depression. Anhui Medical and Pharmaceutical Journal, 9(11): 801-803. 